\title{
THE MAXIMAL QUOTIENT RING OF REGULAR GROUP RINGS. II
}

\author{
FERRAN CEDÓ
}

(Communicated by Donald S. Passman)

\begin{abstract}
Let $K$ be a commutative field. Let $G$ be a locally finite group without elements of order $p$ in case char $K=p>0$. In this paper it is proved that for some large classes of groups $G$ ( $\Delta$-hypercentral, residually finite and groups satisfying Min- $q$ for all primes $q$ ) the Type $\mathrm{I}_{\infty}$ part of the maximal quotient ring of the group ring $K[G]$ is zero.
\end{abstract}

Introduction. Let $K$ be a (commutative) field and let $G$ be a group. Recall that the group ring $K[G]$ is (von Neumann) regular if and only if $G$ is locally finite and has no elements of order $p$ in case char $K=p>0$. For the notation and general theory on group rings, cf. [10].

Recall that the maximal right quotient ring $Q^{r}(K[G])$ of a regular group ring $K[G]$ is uniquely a direct product of rings of Types $\mathrm{I}_{f}, \mathrm{I}_{\infty}, \mathrm{II}_{f}, \mathrm{II}_{\infty}$ and III (see [3, Theorem 10.22]). We are interested in this decomposition, specially in the following questions: If $K[G]$ is regular, is the Type $\mathrm{I}_{\infty}$ part of $Q^{r}(K[G])$ zero? And the Type II part?

In [4] Goursaud and Valette answer in the positive the first question for some special cases, namely when $K$ either has positive characteristic or contains all roots of unity. The question of whether this is true for arbitrary fields remains open. We answer this question for some classes of groups, namely when $G$ is either $\Delta$ hypercentral, residually finite or satisfies Min- $p$ for all primes $p$. While our results here are by no means definitive, we hope that some of our techniques constitute a first step towards the complete solution of this problem.

As far as concerns the second question, Hannah $[\mathbf{5}, \mathbf{6}]$ gives some partial answers by using countable techniques. By using Hannah's results we can answer the question in the positive whether the group $G$ is $\Delta$-hypercentral.

I am indebted to Professor B. Hartley for his stimulating letters on locally finite groups, from which I was aware of Theorem 2.3 below.

1. $\Delta$-hypercentral groups. Let $G$ be a group. Recall that $\Delta(G)=\{g \in$ $\left.G \mid\left[G: C_{G}(g)\right]<\infty\right\}$ is a characteristic subgroup of $G$. We define the upper $\Delta$ central series $\left\{\Delta_{\alpha}\right\}$ of $G$ by setting

$$
\begin{aligned}
& \Delta_{0}(G)=\langle 1\rangle, \\
& \Delta_{\alpha+1}(G) / \Delta_{\alpha}(G)=\Delta\left(G / \Delta_{\alpha}(G)\right), \\
& \Delta_{\beta}(G)=\bigcup_{\alpha<\beta} \Delta_{\alpha}(G)
\end{aligned}
$$

Received by the editors October 8, 1987.

1980 Mathematics Subject Classification (1985 Revision). Primary 16A08, 16A27, 16 A30.

This work was partially supported by CAICYT Grant 3556/83. 
for each ordinal $\alpha$ and limit ordinal $\beta$. We call the last term in this series the $\Delta$-hypercentre of $G$, and we denote it by $\Delta_{\infty}(G)$. If $G=\Delta_{\infty}(G)$ we say that $G$ is $\Delta$-hypercentral.

LEMMA 1.1. Let $R$ be a regular ring. Let $M$ be a right $R$-module. Then the natural homomorphism End $M \rightarrow \prod \operatorname{End}(M / M P)$, where the product is over all prime ideals $P$ of $R$, is an injection.

Proof. Set $N=\bigcap_{P} M P$, now $N P=N$ for all prime ideals $P$ of $R$. By $[\mathbf{3}$, Lemma 6.14], we have $N=0$.

LEMMA 1.2. Let $R$ be a regular ring. Set

$$
I=\{x \in R \mid \operatorname{End}(x R) \text { has bounded index of nilpotence }\} \text {. }
$$

Then $I$ is an ideal of $R$.

ProOf. Let $x \in I$ and $r \in R$, we shall see that $x r, r x \in I$. Note that $x r R$ is a direct summand of $x R$. Hence any $\varphi \in \operatorname{End}(x r R)$ can be extended to $\bar{\varphi} \in \operatorname{End}(x R)$ and if $\varphi$ is nilpotent, $\bar{\varphi}$ can be chosen nilpotent. Thus $x r \in I$. In order to prove that $r x \in I$, we may assume that $x^{2}=x$. Now $\operatorname{End}(x R) \cong x R x \cong \operatorname{End}(R x)$ and by symmetry $r x \in I$.

Let $x, y \in I$, we shall see that $x+y \in I$. Let $e, f \in R$ be idempotents such that $x R=e R$ and $y R=f R$. Then $e, f \in I$. Set $M=e R+f R$. By the proof of $[3$, Theorem 1.1], we may assume that $e f=f e=0$. Let $n$ be a positive integer such that the indices of nilpotence of $e R e$ and $f R f$ are $\leq n$. Set $\bar{R}=R / P$, where $P$ is a prime ideal of $R$. Now $M / M P \cong \bar{e} \bar{R} \oplus \bar{f} \bar{R}$. By [3, Lemma 7.6], the indices of nilpotence of $\bar{e} \bar{R} \bar{e}$ and $\bar{f} \bar{R} \bar{f}$ are $\leq n$. By [3, Theorem 7.9], $\bar{e} \bar{R} \bar{e} \cong M_{m}(D)$ and $\bar{f} \bar{R} \bar{f} \cong M_{m^{\prime}}\left(D^{\prime}\right)$, where $m, m^{\prime} \leq n$ and $D$ and $D^{\prime}$ are division rings. Thus End $(\bar{e} \bar{R} \oplus \bar{f} \bar{R})$ has index of nilpotence $m+m^{\prime} \leq 2 n$. Now, by Lemma 1.1 , it is easy to see that $\operatorname{End}(e R \oplus f R)$ has bounded index of nilpotence, thus $e R \oplus f R \subseteq I$ and $x+y \in I$.

THEOREM 1.3. Let $K[G]$ be a regular group ring. Suppose that $G$ is $\Delta$ hypercentral. Then the Type $\mathrm{I}_{\infty}$ part of $Q^{r}(K[G])$ is zero.

ProOF. Suppose that the Type I part of $Q^{r}(K[G])$ is nonzero. Consider $I=$ $\{\alpha \in K[G] \mid \operatorname{End}(\alpha K[G])$ has bounded index of nilpotence $\}$. Then, by Lemma 1.2, $I$ is an ideal of $K[G]$, and since the Type I part of $Q^{r}(K[G])$ is nonzero, $I \neq 0$. By $[10$, Theorem 8.4.10], $I \cap K[\Delta(G)] \neq 0$.

By [4, Théorème 1.3], we may assume that char $K=0$. Let $e \in I \cap K[\Delta(G)]$ be a nonzero idempotent. Set $H=C_{G}(\operatorname{Supp} e)$. By [10, Theorem 2.1.8], $\pi_{H}(e) \neq$ 0 . Now there exists $\beta \in K\left[\left\langle\operatorname{Supp} \pi_{H}(e)\right\rangle\right]$ such that $f=\pi_{H}(e) \beta$ is a nonzero idempotent. Clearly $f$ is a central idempotent of $K[H]$. Suppose that $e K[G] e$ has index $n$. We shall see that $f K[H]$ has index $\leq n$. Suppose that $(f \alpha)^{m}=0$, for certain $\alpha \in K[H]$. Then $(e f \alpha)^{m}=e(f \alpha)^{m}=0$, and so $(e f \alpha)^{n}=e(f \alpha)^{n}=$ 0 . Hence $\pi_{H}(e) \beta f \alpha^{n}=0$ and $(f \alpha)^{n}=0$. Thus $f K[H]$ has bounded index of nilpotence. By [3, Corollary 7.4], $f Q^{r}(K[H])$ has bounded index of nilpotence. By [3, Theorems 7.20 and 10.24], $f Q^{r}(K[H])$ is Type $\mathrm{I}_{f}$. By [2, Theorem 2.3], $[H: \Delta(H)]<\infty$ and $\left|\Delta(H)^{\prime}\right|<\infty$. Since $[G: H]<\infty$, we have $[G: \Delta(G)]<\infty$ and $\left|\Delta(G)^{\prime}\right|<\infty$. Now it is easy to see that $G$ has a nilpotent subgroup of finite 
index. By [6, Theorem 6.1] and [2, Proposition 1.1], the Type I part of $Q^{r}(K[G])$ is Type $\mathrm{I}_{f}$ bounded.

Let $R$ be a ring. If a right ideal $I$ of $R$ is essential in $R$, we write $I \leq_{e} R$.

LEMMA 1.4. Let $K[G]$ be a nonsingular group ring. Let $H$ be a subgroup of $G$ such that $[\langle H \cup S\rangle: H]<\infty$ and $K[\langle H \cup S\rangle]$ is nonsingular for all finite subsets $S$ of $G$. Then

(i) $I \leq_{e} K[H] \Rightarrow I K[G] \leq_{e} K[G]$,

(ii) $Q^{r}(K[H]) \subseteq Q^{r}(K[G])$.

ProOF. (i) Suppose that $I \leq_{e} K[H]$. Let $\alpha$ be a nonzero element of $K[G]$. Set $G_{1}=\langle H \cup \operatorname{Supp} \alpha\rangle$. Since $\left[G_{1}: H\right]<\infty$, by [9, Corollary 13], we have $Q^{r}\left(K\left[G_{1}\right]\right) \cong K\left[G_{1}\right] \otimes_{K[H]} Q^{r}(K[H])$. Since $K\left[G_{1}\right]$ is a free $K[H]$-module and $Q^{r}(K[H])$ is a right nonsingular $K[H]$-module, we have that $Q^{r}\left(K\left[G_{1}\right]\right)$ is a right nonsingular $K[H]$-module. Hence $l_{Q^{r}\left(K\left[G_{1}\right]\right)}\left(I Q^{r}\left(K\left[G_{1}\right]\right)\right)=0$. Since $Q^{r}\left(K\left[G_{1}\right]\right)$ is right self-injective, $I Q^{r}\left(K\left[G_{1}\right]\right) \leq_{e} Q^{r}\left(K\left[G_{1}\right]\right)$. Thus $I K[G] \cap \alpha K[G] \neq 0$ and so $I K[G] \leq_{e} K[G]$.

(ii) By (i) it is easy to see that $Q^{r}(K[G])$ is a right nonsingular $K[H]$-module. Since $Q^{r}(K[G])$ is a right self-injective $K[G]$-module and $K[G]$ is a free $K[H]$ module, $Q^{r}(K[G])$ is a right self-injective $K[H]$-module. Thus $\left\{x \in Q^{r}(K[G]) \mid\right.$ $\left.(x: K[H]) \leq_{e} K[H]\right\}$ is the maximal right quotient ring of $K[H]$.

Let $G$ be a group. Recall that $\Delta^{+}(G)=\{x \in \Delta(G) \mid o(x)<\infty\}$ is a characteristic subgroup of $G$. As above we may define the $\Delta^{+}$-central series $\left\{\Delta_{\alpha}^{+}\right\}$of $G$ and $\Delta_{\infty}^{+}(G)$, the last term in this series.

LEMMA 1.5. Let $G$ be a group and let $H$ be a subgroup of $G$. Suppose that $K[N]$ is a nonsingular group ring for all subgroups $N$ of $G$. Then

(i) $I \leq_{e} K[H] \Rightarrow I K\left[\Delta_{\infty}^{+}(G) H\right] \leq_{e} K\left[\Delta_{\infty}^{+}(G) H\right]$,

(ii) $Q^{r}(K[H]) \subseteq Q^{r}\left(K\left[\Delta_{\infty}^{+}(G) H\right]\right)$.

ProOF. (i) Let $\alpha$ be an ordinal. By transfinite induction, we shall see that $I \leq_{e}$ $K[H] \Rightarrow I K\left[\Delta_{\alpha}^{+}(G) H\right] \leq_{e} K\left[\Delta_{\alpha}^{+}(G) H\right]$. If $\alpha=0$, then the result is clear. Suppose that $\alpha>0$ is not a limit ordinal and that $I K\left[\Delta_{\alpha-1}^{+}(G) H\right] \leq_{e} K\left[\Delta_{\alpha-1}^{+}(G) H\right]$. Consider $\bar{G}=\Delta_{\alpha}^{+}(G) H / \Delta_{\alpha-1}^{+}(G)$ and $\bar{H}=\Delta_{\alpha-1}^{+}(G) H / \Delta_{\alpha-1}^{+}(G)$. Now $\bar{G}=$ $\Delta^{+}(\bar{G}) \bar{H}$ and it is easy to see that $[\langle\bar{H} \cup S\rangle: \bar{H}]<\infty$ for all finite subsets $S$ of $\bar{G}$. By Lemma 1.4, we have $I K\left[\Delta_{\alpha}^{+}(G) H\right] \leq_{e} K\left[\Delta_{\alpha}^{+}(G) H\right]$. Now suppose that $\alpha$ is a limit ordinal and that $I K\left[\Delta_{\beta}^{+}(G) H\right] \leq_{e} K\left[\Delta_{\beta}^{+}(G) H\right]$ for all $\beta<\alpha$. Let $x$ be a nonzero element of $K\left[\Delta_{\alpha}^{+}(G) H\right]$. There exists an ordinal $\beta<\alpha$ such that $x \in K\left[\Delta_{\beta}^{+}(G) H\right]$. Hence $I K\left[\Delta_{\beta}^{+}(G) H\right] \cap x K\left[\Delta_{\beta}^{+}(G) H\right] \neq 0$ and so $I K\left[\Delta_{\alpha}^{+}(G) H\right] \cap x K\left[\Delta_{\alpha}^{+}(G) H\right] \neq 0$.

(ii) It follows as the proof of Lemma 1.4.

THEOREM 1.6. Let $K[G]$ be a regular group ring. Suppose that $G$ is $\Delta$ hypercentral. Then the Type II part of $Q^{r}(K[G])$ is zero.

ProOF. Suppose that the Type II part of $Q^{r}(K[G])$ is nonzero. Then there exists a nonzero idempotent $e \in K[G]$ such that $e Q^{r}(K[G]) e$ is directly finite. Let $H$ be a countable subgroup of $G$ such that Supp $e \subseteq H$. By Lemma 1.5, $e Q^{r}(K[H]) e \subseteq e Q^{r}(K[G]) e$, so $e Q^{r}(K[H]) e$ is directly finite. By [5, Theorem 3.3], the Type I part of $Q^{r}(K[H])$ is nonzero. By Theorem 1.3, the Type $\mathrm{I}_{f}$ part of $Q^{r}(K[H])$ is nonzero. By [2, Theorem 2.3], $[H: \Delta(H)]<\infty$ and $\left|\Delta(H)^{\prime}\right|<\infty$. 
Now it is easy to see that $[G: \Delta(G)]<\infty$ and $\left|\Delta(G)^{\prime}\right|<\infty$. But by [5, Theorem 3.3] and [6, Theorem 6.1], we have that the Type II part of $Q^{r}(K[G])$ is zero, a contradiction, therefore the Type II part of $Q^{r}(K[G])$ is zero.

REMARK. In fact the hypothesis of Theorem 1.6 can be relaxed and, by using the techniques of Hannah's paper [5] plus Lemma 1.5, we can prove that the Type III part of $Q^{r}(K[G])$ is biregular.

2. The Type I part. Next Lemma is an easy consequence of [1, Theorem 11].

LEMMA 2.1. Let $K[G]$ be a regular group ring. Let $e \in K[G]$ be an abelian idempotent. If $f \in e K[G] e$ is an idempotent then Supp $f \subseteq\langle\Delta(G) \cup \operatorname{Supp} e\rangle$.

THEOREM 2.2. Let $K[G]$ be a regular group ring. Suppose that $G$ is residually finite. Then the Type $\mathrm{I}_{\infty}$ part of $Q^{r}(K[G])$ is zero.

ProOF. Suppose that the Type I part of $Q^{r}(K[G])$ is nonzero. We shall see that $[G: \Delta(G)]<\infty$.

Suppose that $[G: \Delta(G)]=\infty$. Let $e \in K[G]$ be a nonzero abelian idempotent and set $H=\langle\operatorname{Supp} e\rangle$. Since $G$ is residually finite, there exists $N \triangleleft G$ such that $[G: N]<\infty$ and $H \cap N=\langle 1\rangle$. Let $g \in N \backslash \Delta(G) H$. Now $e(1-g) \neq 0$, since $\pi_{H}(e(1-g))=e \neq 0$. Since $K[G]$ is regular, there exists $\beta \in K[G]$ such that

$$
e(1-g) \beta e(1-g)=e(1-g) \text {. }
$$

Hence

$$
(e(1-g) \beta e)^{2}=e(1-g) \beta e(1-g) \beta e=e(1-g) \beta e
$$

is an idempotent of $e K[G] e$. By Lemma 2.1, $e(1-g) \beta e \in K[\Delta(G) H]$. Hence

$$
e=\pi_{\Delta(G) H}(e(1-g))=\pi_{\Delta(G) H}(e(1-g) \beta e(1-g))=e(1-g) \beta e .
$$

Now $\rho_{N}(e)=\rho_{N}(e(1-g) \beta e)=0$, but $H \cap N=\langle 1\rangle$, a contradiction, thus $[G: \Delta(G)]<\infty$. The result follows by Theorem 1.3.

Next theorem is a well-known consequence of the classification of finite simple groups. We give a proof for the sake of completeness.

THEOREM 2.3. Let $G$ be a locally finite group. Suppose that $G$ satisfies Min-p for all primes $p$. Then $G$ has a locally soluble subgroup of finite index.

Proof. Suppose that $G$ involves a countable infinite, locally finite simple group $H$. By [8, Theorem 3.13], $H$ satisfies Min- $p$ for all primes $p$. By [8, Proposition 4.7] and the classification of finite simple groups, there exists a field $F$ of finite characteristic and a natural number $n$ such that the group $H$ is isomorphic to a subgroup of $G L(n, F)$. Now by [8, Theorem 1.L.4], $H$ is finite, a contradiction. Thus, by [8, Theorem 4.4], $G$ does not involve any infinite simple group. The result follows by [8, Corollary 3.18].

Let $G$ be a group. We denote by $\pi(G)$ the set of all primes $p$ such that $G$ has an element of order $p$. If $H$ is a finite subgroup of $G$, we denote by $\hat{H}$ the sum of the finitely many elements of $H$ in $K[G]$.

THEOREM 2.4. Let $K[G]$ be a regular group ring. Suppose that $G$ is countable and $G / \Delta_{\infty}(G)$ satisfies Min-p for all primes $p$. Then the Type $\mathrm{I}_{\infty}$ part of $Q^{r}(K[G])$ is zero.

Proof. Suppose that the Type I part of $Q^{r}(K[G])$ is nonzero. We shall see that $G=\Delta_{\infty}(G)$. 
Let $e \in K[G]$ be a nonzero abelian idempotent. Set $G_{1}=\left\langle\Delta_{\infty}(G) \cup \operatorname{Supp} e\right\rangle$. Then the Type I part of $Q^{r}\left(K\left[G_{1}\right]\right)$ is nonzero. By Theorem 1.3, the Type $I_{f}$ part of $Q^{r}\left(K\left[G_{1}\right]\right)$ is nonzero. Let $M$ be the smallest normal subgroup of $\Delta_{\infty}(G)$ with $\Delta_{\infty}(G) / M$ abelian-by-finite. By [2, Theorem 2.3], $M$ exists and it is a finite characteristic subgroup of $G$. By [2, Theorem 2.3] and [6, Theorem 6.1] $\hat{M} /|M| Q^{r}\left(K\left[G_{1}\right]\right)$ is the Type I part of $Q^{r}\left(K\left[G_{1}\right]\right)$. Hence $e \in \hat{M} /|M| K[G] \cong K[G / M]$ and so the Type I part of $Q^{r}(K[G / M])$ is nonzero. Thus we may assume that $\Delta_{\infty}(G)$ is abelian-by-finite. By [10, Lemma 12.1.2], $\Delta_{\infty}(G)$ has a characteristic abelian subgroup $A$ of finite index.

Now $A$ is the direct product $A=\prod A_{p}$ of its $p$-primary parts. Set $\pi=$ $\pi(\langle\operatorname{Supp} e\rangle)$ and $A_{\pi^{\prime}}=\prod_{p \notin \pi} A_{p}$. Then $A_{\pi^{\prime}}$ is a normal subgroup of $G$. Consider $\bar{G}=G / A_{\pi^{\prime}}$, then, by [3, Lemma 7.6], $\bar{e} \in K[\bar{G}]$ is an abelian idempotent and clearly it is nonzero, so the Type I part of $Q^{r}(K[\bar{G}])$ is nonzero. Thus we may assume that $\pi\left(\Delta_{\infty}(G)\right)=\sigma$ is finite.

By Theorem 2.3 and [6, Theorem 6.1], we may assume that $G$ is locally soluble. Set $\widetilde{G}=G / \Delta_{\infty}(G)$. By $[\mathbf{8}$, Corollary 3.18], $\widetilde{G}$ has an abelian normal subgroup $\widetilde{B}$ such that $\widetilde{G} / \widetilde{B}$ is residually finite and every Sylow subgroup of $\widetilde{G} / \widetilde{B}$ is finite. Since $\Delta(\widetilde{G})=\langle 1\rangle$ it is easy to see that $\widetilde{B}=\langle 1\rangle$, so $\widetilde{G}$ is residually finite and every Sylow subgroup of $\widetilde{G}$ is finite.

For each $p \in \sigma$, let $\widetilde{S}_{p}$ be a Sylow $p$-subgroup of $\widetilde{G}$. Set $\widetilde{S}=\left\langle\widetilde{S}_{p} \mid p \in \sigma\right\rangle$, then $\widetilde{S}$ is a finite subgroup of $\widetilde{G}$. Since $\widetilde{G}$ is residually finite, there exists a normal subgroup $\widetilde{N}$ of $\widetilde{G}$ of finite index such that $\widetilde{N} \cap \widetilde{S}=\langle 1\rangle$. By [8, Theorem, p. 190] it is easy to see that $\tilde{N}$ is a $\sigma^{\prime}$-group. By $\left[\mathbf{1 0}\right.$, Lemma 12.4.12], there exists a $\sigma^{\prime}$-subgroup $N$ of $G$ such that $N \Delta_{\infty}(G) / \Delta_{\infty}(G)=\tilde{N}$. Hence $N \cong \tilde{N}$ and $\Delta(N)=\langle 1\rangle$. Since $\Delta_{\infty}(G) N$ is a subgroup of $G$ of finite index, we have, by [6, Theorem 6.1], that the Type I part of $Q^{r}\left(K\left[\Delta_{\infty}(G) N\right]\right)$ is nonzero. Let $\beta$ be the smallest ordinal such that the Type I part of $Q^{r}\left(K\left[\Delta_{\beta}(G) N\right]\right)$ is nonzero. Let $f \in K\left[\Delta_{\beta}(G) N\right]$ be a nonzero abelian idempotent. It is clear that $\beta$ is not a limit ordinal. Suppose that $\beta \neq 0$. Now $\left[\left\langle\Delta_{\beta-1}(G) N \cup \operatorname{Supp} f\right\rangle: \Delta_{\beta-1}(G) N\right]<\infty$ and, by [6, Theorem 6.1], the Type I part of $Q^{r}\left(K\left[\Delta_{\beta-1}(G) N\right]\right)$ is nonzero, a contradiction, thus $\beta=0$ and the Type I part of $Q^{r}(K[N])$ is nonzero. Since $K[N]$ is prime and $N$ is countable we have, by [7, Theorem 2.3], that $N=\langle 1\rangle$. Hence $\Delta_{\infty}(G)=\Delta_{\infty}(G) N$. Since $\left[G: \Delta_{\infty}(G) N\right]<\infty$, we have $G=\Delta_{\infty}(G)$. Now by Theorem 1.3 we obtain the result.

PROPOSITION 2.5. Let $K[G]$ be a regular group ring. Suppose that $G$ is countable and locally soluble. If the Type I part of $Q^{r}(K[G])$ is nonzero, then $G / \Delta_{\infty}(G)$ satisfies Min-p for almost all primes $p$.

ProOF. Let $e \in K[G]$ be a nonzero abelian idempotent. Set $\pi=\pi(\langle\operatorname{Supp} e\rangle)$. As in proof of Theorem 2.4, we may assume that $\Delta_{\infty}(G)$ is abelian-by-finite and $\pi\left(\Delta_{\infty}(G)\right)=\sigma$ is finite. Let $p$ be a prime such that $p \notin \pi \cup \sigma$. Set $\tau=\pi \cup \sigma \cup$ $\{p\}$. Since $G$ is a countable locally soluble group, by using the theory of the Hall subgroups, it is easy to construct a $\tau$-subgroup $G_{1}$ of $G$ such that:

(i) $\langle$ Supp $e\rangle \subseteq G_{1}$.

(ii) If $F$ is a finite $\tau$-subgroup of $G$, then there exists $g \in G$ such that $F^{g} \subseteq G_{1}$. 
We shall see that all $p$-subgroups of $G_{1}$ are finite. By (i), the Type I part of $Q^{r}\left(K\left[G_{1}\right]\right)$ is nonzero. Since $\pi\left(G_{1}\right)$ is finite we have, by [2, Proposition 1.2] that the Type I part of $Q^{r}\left(K\left[G_{1}\right]\right)$ is $\hat{M} /|M| Q^{r}\left(K\left[G_{1}\right]\right)$, where $M$ is the smallest normal subgroup of $G_{1}$ such that $G_{1} / M$ is abelian-by-finite. So we may assume that $G_{1}$ is abelian-by-finite. Let $A$ be an abelian normal subgroup of $G_{1}$ of finite index. Suppose that there exists an infinite $p$-subgroup $P$ of $G_{1}$. Then $Q=A \cap P \neq\langle 1\rangle$. Let $g \in Q \backslash\langle 1\rangle$. Consider $B=\left\langle g^{x} \mid x \in\langle\operatorname{Supp} e\rangle\right\rangle$. Then $B$ is a finite $p$-subgroup of $A$. Since $p \notin \pi, e \hat{B} /|B|$ is a nonzero idempotent of $e K\left[G_{1}\right] e$. By Lemma 2.1, Supp $e \hat{B} /|B| \subseteq \Delta(G)\langle\operatorname{Supp} e\rangle$. Now $(1-g) e \hat{B} /|B|=(1-g) \hat{B} e /|B|=0$, but $g$ is a $p$-element and $p \notin \pi \cup \sigma$, a contradiction, thus all $p$-subgroups of $G_{1}$ are finite.

Now by (ii), all $p$-subgroups of $G$ are finite.

\section{REFERENCES}

1. E. P. Armendariz, H. K. Koo and J. K. Park, Compressible group algebra, Comm. Algebra 13 (1985), 1763-1777.

2. F. Ced6, On the maximal quotient ring of regular group rings, J. Algebra 115 (1988), 164-174.

3. K. R. Goodearl, Von Neumann regular rings, Pitman, London, 1979.

4. J.-M. Goursaud et J. Valette, Sur l'enveloppe injective des anneaux de groupes réguliers, Bull. Soc. Math. France 103 (1975), 91-102.

5. J. Hannah, Countability in regular self-injective rings, Quart. J. Math. Oxford 31 (1980), 315327.

6. _ Ideals in regular self-injective rings and quotient rings of group algebras, Proc. London Math. Soc. 42 (1981), 533-558.

7. J. Hannah and K. C. O'Meara, Maximal quotient rings of prime group algebras, Proc. Amer. Math. Soc. 65 (1977), 1-7.

8. O. H. Kegel and B. A. F. Wehrfritz, Locally finite groups, North-Holland, Amsterdam, London, 1973.

9. K. Louden, Maximal quotient rings of ring extensions, Pacific J. Math. 62 (1976), 489-496.

10. D. S. Passman, The algebraic structure of group rings, Wiley-Interscience, New York, 1977.

Department de MAtemàtiques, Universitat Autònoma de Barcelona, 08193 Bellaterra, Barcelona, SPAin 